\title{
Differentiable Preinvex and Prequasiinvex Functions
}

\author{
Huriye Kadakal $^{1}$ \\ ${ }^{1}$ Ministry of Education, Hamdi Bozba $\breve{g}$ Anatolian High School, Giresun, Turkey
}

\section{Article Info}

Keywords: Invex set, Preinvex function, Prequasiinvex function, Integral inequalities.

2010 AMS: 26A51, 26D10, $26 D 15$.

Received: 20 November 2019

Accepted: 11 May 2020

Available online: 22 June 2020

\begin{abstract}
In this paper, a new identity for functions defined on an open invex subset of set of real numbers is established, and by using this identity and the Hölder and Power mean integral inequalities we present new integral inequalities for functions whose powers of derivatives in absolute value are preinvex and prequasiinvex. We should especially mention that the results obtained in special cases coincide with the well-known results in the literature.
\end{abstract}

\section{Introduction}

Definition 1.1. A function $f: I \subseteq \mathbb{R} \rightarrow \mathbb{R}$ is said to be convex if the inequality $f(t x+(1-t) y) \leq t f(x)+(1-t) f(y)$ is valid for all $x, y \in I$ and $t \in[0,1]$. If this inequality reverses, then $f$ is said to be concave on interval $I \neq \varnothing$.

This definition is well known in the literature. Convexity theory has appeared as a powerful technique to study a wide class of unrelated problems in pure and applied sciences.

Let $f: I \subseteq \mathbb{R} \rightarrow \mathbb{R}$ be a convex function on the interval $I$ of real numbers and $a, b \in I$ with $a<b$. The celebrated double inequality

$$
f\left(\frac{a+b}{2}\right) \leq \frac{1}{b-a} \int_{a}^{b} f(x) d x \leq \frac{f(a)+f(b)}{2},
$$

is well known in the literature as Hermite-Hadamard's integral inequality for convex functions [22]. Both inequalities hold in the reserved direction if the function $f$ is concave. The classical Hermite-Hadamard integral inequality provides estimates of the mean value of a continuous convex or concave function. Hadamard's integral inequality for convex or concave functions has received renewed attention in recent years and a remarkable variety of refinements and generalizations have been found; for example see $[4,9,10,18,21,22,24]$. Hermite-Hadamard (H-H) integral inequality (see [5]) has been considered the most useful inequality in mathematical analysis. Some of the classical inequalities for means can be derived from the above $\mathrm{H}-\mathrm{H}$ inequality for particular choices of the function $f$.

Definition 1.2. A function $f: I \subseteq \mathbb{R} \rightarrow \mathbb{R}$ is said to be quasi-convex if the inequality $f(t x+(1-t) y) \leq \max \{f(x), f(y)\}$ holds for all $x, y \in I$ and $t \in[0,1]$. Clearly, any convex function is a quasi-convex function. Furthermore, there exist quasi-convex functions which are not convex [6].

Let us recall the notions of preinvexity and prequasiinvexity which are signicant generalizations of the notions of convexity and quasiconvexity respectively, and some related results.

Definition 1.3 ( [25]). Let $K$ be a non-empty subset in $\mathbb{R}^{n}$ and $\eta: K \times K \rightarrow \mathbb{R}^{n}$. Let $x \in K$, then the set $K$ is said to be invex at $x$ with respect to $\eta(\cdot, \cdot)$, if $x+t \eta(y, x) \in K, \quad \forall x, y \in K \quad t \in[0,1] . K$ is said to be an invex set with respect to $\eta$ if $K$ is invex at each $x \in K$. The invex set $K$ is also called $\eta$-connected set.

This definition essentially says that there is a path starting from a point $x$ which is contained in $K$. We do not require that the point $y$ should be one of the end points of the path. This observation plays an important role in our analysis. Note that, if we demand that $y$ should be an end point of the path for every pair of points $x, y \in K$, then $\eta(y, x)=y-x$, and consequently invexity reduces to convexity [1]. Thus, it is true that every convex set is also an invex set with respect to $\eta(y, x)=y-x$, but the converse is not necessarily true, see [14,26] and the references therein. For the sake of simplicity, we always assume that $K=[x, x+t \eta(y, x)]$, unless otherwise specified. 
Definition 1.4 ( [25]). A function $f: K \rightarrow \mathbb{R}$ on an invex set $K \subseteq \mathbb{R}$ is said to be preinvex with respect to $\eta$, if

$$
f(u+t \eta(v, u)) \leq(1-t) f(u)+t f(v), \quad \forall u, v \in K, \quad t \in[0,1]
$$

The function $f$ is said to be preconcave if and only if $-f$ is preinvex.

It is to be noted that every convex function is preinvex with respect to the map $\eta(y, x)=x-y$ but the converse is not true see for instance.

Definition 1.5 ( [2]). A function $f: K \rightarrow \mathbb{R}$ on an invex set $K \subseteq \mathbb{R}$ is said to be prequasiinvex with respect to $\eta$, if

$$
f(u+t \eta(v, u)) \leq \max \{f(u), f(v)\}, \quad \forall u, v \in K, t \in[0,1] .
$$

Also every quasi-convex function is a prequasiinvex with respect to the map $\eta(v, u)=v-u$ but the converse does not hold, see for example [2].

Mohan and Neogy [14] introduced Condition $C$ as follows:

Definition 1.6 ([14]). Let $S \subseteq \mathbb{R}$ be an open invex subset with respect to the map $\eta: S \times S \rightarrow \mathbb{R}$. We say that the function $\eta$ satisfies the Condition $C$ if, for any $x, y \in S$ and any $t \in[0,1]$,

$$
\begin{aligned}
& \eta(y, y+t \eta(x, y))=-t \eta(x, y) \\
& \eta(x, y+t \eta(x, y))=(1-t) \eta(x, y) .
\end{aligned}
$$

Note that, from the Condition $C$, we have

$$
\eta\left(y+t_{2} \eta(x, y), y+t_{1} \eta(x, y)\right)=\left(t_{2}-t_{1}\right) \eta(x, y)
$$

for any $x, y \in S$ and any $t_{1}, t_{2} \in[0,1]$.

In recent years, many mathematicians have been studying about preinvexity and types of preinvexity. These studies include, among others, the work of Ben-Israel and Mond [7], Pini [23], Noor [16, 17], Yang and Li [27] and Weir and Mond [25]. Ben-Israel and Mond, Weir and Mond and Noor have studied the basic properties of the preinvex functions and their role in optimization, variational inequalities and equilibrium problems.

In a recent paper, Noor [15] has obtained the following Hermite-Hadamard type integral inequalities for the preinvex functions.

Theorem 1.7 ( [15]). Let $f:[a, a+\eta(b, a)] \rightarrow(0, \infty)$ be a preinvex function on the interval of the real numbers $K^{\circ}$ (the interior of $\left.K\right)$ and $a, b \in K^{\circ}$ with $\eta(b, a)>0$. Then the following inequalities hold

$$
f\left(\frac{2 a+\eta(b, a)}{2}\right) \leq \frac{1}{\eta(b, a)} \int_{a}^{a+\eta(b, a)} f(x) d x \leq \frac{f(a)+f(b)}{2}
$$

For several recent results on inequalities for preinvex and prequasiinvex functions which are connected to (1.3), we refer the interested reader to $[3,11,13,19,20]$ and the references therein.

Let $0<a<b$, throughout this paper we will use

$$
\begin{aligned}
A(a, b) & =\frac{a+b}{2} \\
L_{p}(a, b) & =\left(\frac{b^{p+1}-a^{p+1}}{(p+1)(b-a)}\right)^{\frac{1}{p}}, a \neq b, p \in \mathbb{R}, p \neq-1,0
\end{aligned}
$$

for the arithmetic and generalized logarithmic means, respectively.

\section{Main results}

In this section, using a general integral identity for a differentiable functions, we establish some new integral inequalities for mappings whose derivative in absolute value at certain powers are preinvex and prequasiinvex. We will use the following Lemma to prove our main results.

Lemma 2.1. Let $K \subseteq \mathbb{R}$ be an open invex subset with respect to mapping $\eta(\cdot, \cdot): K \times K \rightarrow \mathbb{R}^{n}$ and $a, b \in K$ with $\eta(b, a)>0$. Suppose that $f: K \rightarrow \mathbb{R}$ is a differentiable function on $K$ such that $f^{\prime} \in L[a, a+\eta(b, a)]$. Then the following identity holds:

$$
f(a+\eta(b, a))[a+\eta(b, a)]-f(a) a-\int_{a}^{a+\eta(b, a)} f(x) d x=\eta(b, a) \int_{0}^{1}(a+t \eta(b, a)) f^{\prime}(a+t \eta(b, a)) d t .
$$

Proof. By changing the variable and integrating by parts, we have

$$
\begin{aligned}
\eta(b, a) \int_{0}^{1}(a+t \eta(b, a)) f^{\prime}(a+t \eta(b, a)) d t & =\int_{a}^{a+\eta(b, a)} x f^{\prime}(x) d x \\
& =f(a+\eta(b, a))[a+\eta(b, a)]-f(a) a-\int_{a}^{a+\eta(b, a)} f(x) d x .
\end{aligned}
$$

This completes the proof of lemma. 
Theorem 2.2. Let $K \subseteq \mathbb{R}$ be an open invex subset with respect to mapping $\eta(\cdot, \cdot): K \times K \rightarrow \mathbb{R}^{n}$ and $a, b \in K$ with $\eta(b, a)>0$. Suppose that $f: K \rightarrow \mathbb{R}$ is a differentiable function on $K$ such that $f^{\prime} \in L[a, a+\eta(b, a)]$. If $\left|f^{\prime}\right|^{q}$ is preinvex on $K$ for $q>1$, then the following inequality holds:

$$
\left|f(a+\eta(b, a))[a+\eta(b, a)]-f(a) a-\int_{a}^{a+\eta(b, a)} f(x) d x\right| \leq \eta^{1 / q}(b, a) C_{\eta}^{\frac{1}{p}}(p, a, b) A^{\frac{1}{q}}\left(\left|f^{\prime}(a)\right|^{q},\left|f^{\prime}(b)\right|^{q}\right) .
$$

where,

$$
C_{\eta}(p, a, b):= \begin{cases}\eta(b, a) L_{p}^{p}[a+\eta(b, a), a], & a>0, a+\eta(b, a)>0 \\ \frac{2}{p+1} A\left((a+\eta(b, a))^{p+1},(-a)^{p+1}\right), & a<0, a+\eta(b, a)>0 \\ \eta(b, a) L_{p}^{p}[-a,-(a+\eta(b, a))], & a<0, a+\eta(b, a)<0 .\end{cases}
$$

Proof. If $\left|f^{\prime}\right|^{q}$ is preinvex on $[a, a+\eta(b, a)]$ for $q>1$, using Lemma 2.1, the Hölder integral inequality and the inequality $\left|f^{\prime}(a+t \eta(b, a))\right|^{q} \leq$ $t\left|f^{\prime}(b)\right|^{q}+(1-t)\left|f^{\prime}(a)\right|^{q}$, we have

$$
\begin{aligned}
\left|f(a+\eta(b, a))[a+\eta(b, a)]-f(a) a-\int_{a}^{a+\eta(b, a)} f(x) d x\right| & \leq \eta(b, a) \int_{0}^{1}|(a+t \eta(b, a))|\left|f^{\prime}(a+t \eta(b, a))\right| d t \\
& \leq \eta(b, a)\left(\int_{0}^{1}|a+t \eta(b, a)|^{p} d t\right)^{\frac{1}{p}}\left(\int_{0}^{1}\left|f^{\prime}(a+t \eta(b, a))\right|^{q} d t\right)^{\frac{1}{q}} \\
& \leq \eta^{\frac{1}{q}}(b, a)\left(\int_{a}^{a+\eta(b, a)}|x|^{p} d x\right)^{\frac{1}{p}}\left(\frac{\left|f^{\prime}(a)\right|^{q}+\left|f^{\prime}(b)\right|^{q}}{2}\right)^{\frac{1}{q}} \\
& =\eta^{\frac{1}{q}}(b, a) C_{\eta}^{\frac{1}{p}}(p, a, b) A^{\frac{1}{q}}\left(\left|f^{\prime}(a)\right|^{q},\left|f^{\prime}(b)\right|^{q}\right) .
\end{aligned}
$$

This completes the proof of theorem.

Corollary 2.3. Suppose that all the assumptions of Theorem 2.2 are satisfied. If we choose $\eta(b, a)=b-a$ then when $\left|f^{\prime}\right|^{q}$ is convex on $K$ for $q>1$ we have

$$
\left|\frac{f(b) b-f(a) a}{b-a}-\frac{1}{b-a} \int_{a}^{b} f(x) d x\right| \leq(b-a)^{\frac{1}{q}-1} C^{\frac{1}{p}}(p, a, b) A^{\frac{1}{q}}\left(\left|f^{\prime}(a)\right|^{q},\left|f^{\prime}(b)\right|^{q}\right) .
$$

where,

$$
C(p, a, b)= \begin{cases}(b-a) L_{p}^{p}(a, b), & a>0, b>0 \\ \frac{2}{p+1} A\left(b^{p+1},(-a)^{p+1}\right), & a<0, b>0, \\ (b-a) L_{p}^{p}(-a,-b), & a<0, b<0 .\end{cases}
$$

We note that this result coincides with Corollary 2.3. in the special case $a, b>0$ [12].

Remark 2.4. If the mapping $\eta$ satisfies condition $C$ then by using of the preinvexity of $\left|f^{\prime}\right|^{q}$ we get

$$
\left|f^{\prime}(a+t \eta(b, a))\right|^{q}=\left|f^{\prime}(a+\eta(b, a)+(1-t) \eta(a, a+\eta(b, a)))\right|^{q} \leq t\left|f^{\prime}(a+\eta(b, a))\right|^{q}+(1-t)\left|f^{\prime}(a)\right|^{q} .
$$

for every $t \in[0,1]$.

If we use the inequality (2.2) in the proof of Theorem 2.2, then the inequality (2.1) becomes to the following inequality:

$$
\left|f(a+\eta(b, a))[a+\eta(b, a)]-f(a) a-\int_{a}^{a+\eta(b, a)} f(x) d x\right| \leq \eta^{\frac{1}{q}}(b, a) C_{\eta}^{\frac{1}{p}}(p, a, b) A^{\frac{1}{q}}\left(\left|f^{\prime}(a)\right|^{q},\left|f^{\prime}(a+\eta(b, a))\right|^{q}\right) .
$$

We note that by using of the preinvexity of $\left|f^{\prime}\right|^{q}$ we have $\left|f^{\prime}(a+\eta(b, a))\right|^{q} \leq\left|f^{\prime}(b)\right|^{q}$.Therefore, the inequality (2.3) is better than the inequality (2.1).

Theorem 2.5. Let $K \subseteq \mathbb{R}$ be an open invex subset with respect to mapping $\eta(\cdot, \cdot): K \times K \rightarrow \mathbb{R}^{n}$ and $a, b \in K$ with $\eta(b, a)>0$. Suppose that $f: K \rightarrow \mathbb{R}$ is a differentiable function on $K$ such that $f^{\prime} \in L[a, a+\eta(b, a)]$. If $\left|f^{\prime}\right|^{q}$ is preinvex on $K$ for $q \geq 1$, then the following inequality holds:

$$
\left|f(a+\eta(b, a))[a+\eta(b, a)]-f(a) a-\int_{a}^{a+\eta(b, a)} f(x) d x\right| \leq \eta^{-\frac{1}{q}}(b, a) D_{1, \eta}^{1-\frac{1}{q}}(a, b)\left[\left|f^{\prime}(b)\right|^{q} D_{2, \eta}(a, b)+\left|f^{\prime}(a)\right|^{q} D_{3, \eta}(a, b)\right]^{\frac{1}{q}}
$$

where

$$
\begin{aligned}
& D_{1, \eta}(a, b):= \begin{cases}\eta(b, a) A(a, a+\eta(b, a)), & a>0, a+\eta(b, a)>0 \\
A\left(a^{2},[a+\eta(b, a)]^{2}\right), & a<0, a+\eta(b, a)>0 \\
-\eta(b, a) A(a, a+\eta(b, a)), & a<0, a+\eta(b, a)<0\end{cases} \\
& D_{2, \eta}(a, b):= \begin{cases}\frac{\eta(b, a)}{6}\left[(2 a+\eta(b, a))(2 \eta(b, a)-a)+2 a^{2}\right], & a>0, a+\eta(b, a)>0 \\
\frac{1}{6}\left[(a+\eta(b, a))^{2}(2 \eta(b, a)-a)-a^{3}\right], & a<0, a+\eta(b, a)>0 \\
-\frac{\eta^{2}(b, a)}{6}(3 a+2 \eta(b, a)), & a<0, a+\eta(b, a)<0\end{cases}
\end{aligned}
$$


and

$$
D_{3, \eta}(a, b):= \begin{cases}\frac{\eta(b, a)}{6}\left[(a+\eta(b, a))(2 a+\eta(b, a))-2 a^{2}\right], & a>0, a+\eta(b, a)>0 \\ \frac{1}{6}\left[(a+\eta(b, a))^{3}+a^{2}(a+3 \eta(b, a))\right], & a<0, a+\eta(b, a)>0 \\ -\frac{\eta^{2}(b, a)}{6}(3 a+\eta(b, a)), & a<0, a+\eta(b, a)<0 .\end{cases}
$$

Proof. From Lemma 2.1 and Power-mean integral inequality, we obtain

$$
\begin{aligned}
& \left|f(a+\eta(b, a))[a+\eta(b, a)]-f(a) a-\int_{a}^{a+\eta(b, a)} f(x) d x\right| \\
& \leq \eta(b, a) \int_{0}^{1}|(a+t \eta(b, a))|\left|f^{\prime}(a+t \eta(b, a))\right| d t \\
& \leq \eta(b, a)\left(\int_{0}^{1}|a+t \eta(b, a)| d t\right)^{1-\frac{1}{q}} \times\left(\int_{0}^{1}|a+t \eta(b, a)|\left|f^{\prime}(a+t \eta(b, a))\right|^{q} d t\right)^{\frac{1}{q}} \\
& \leq \eta^{\frac{1}{q}}(b, a)\left(\int_{a}^{a+\eta(b, a)}|x| d x\right)^{1-\frac{1}{q}} \times\left(\int_{0}^{1}|a+t \eta(b, a)|\left[t\left|f^{\prime}(b)\right|^{q}+(1-t)\left|f^{\prime}(a)\right|^{q}\right] d t\right)^{\frac{1}{q}} \\
& =\eta^{-\frac{1}{q}}(b, a)\left(\int_{a}^{a+\eta(b, a)}|x| d x\right)^{1-\frac{1}{q}}\left(\left|f^{\prime}(b)\right|^{q} \int_{a}^{a+\eta(b, a)}(x-a)|x| d x+\left|f^{\prime}(a)\right|^{q} \int_{a}^{a+\eta(b, a)}[\eta(b, a)-(x-a)]|x| d x\right)^{\frac{1}{q}} \\
& =\eta^{-\frac{1}{q}}(b, a) D_{1, \eta}^{1-\frac{1}{q}}(a, b)\left[\left|f^{\prime}(b)\right|^{q} D_{2, \eta}(a, b)+\left|f^{\prime}(a)\right|^{q} D_{3, \eta}(a, b)\right]^{\frac{1}{q}} .
\end{aligned}
$$

This completes the proof of theorem.

Corollary 2.6. Suppose that all the assumptions of Theorem 2.5 are satisfied. If we choose $\eta(b, a)=b-a$ then when $\left|f^{\prime}\right|^{q}$ is convex on $K$ for $q \geq 1$ we have

$$
\left|\frac{f(b) b-f(a) a}{b-a}-\frac{1}{b-a} \int_{a}^{b} f(x) d x\right| \leq(b-a)^{-1-\frac{1}{q}} D_{1}^{1-\frac{1}{q}}(a, b)\left[\left|f^{\prime}(b)\right|^{q} D_{2}(a, b)+\left|f^{\prime}(a)\right|^{q} D_{3}(a, b)\right]^{\frac{1}{q}},
$$

where

$$
\begin{aligned}
& D_{1}(a, b)=\left\{\begin{array}{lc}
(b-a) A(a, b), & a>0, b>0 \\
A\left(a^{2}, b^{2}\right), & a<0, b>0 \\
-(b-a) A(a, b), & a<0, b<0
\end{array},\right. \\
& D_{2}(a, b)=\left\{\begin{array}{cc}
\frac{1}{6}(b-a)^{2}(2 b+a), & a>0, b>0 \\
\frac{1}{6}\left[b^{2}(2 b-3 a)-a^{3}\right], & a<0, b>0 \\
-\frac{1}{6}(b-a)^{2}(a+2 b), & a<0, b<0
\end{array}\right.
\end{aligned}
$$

and

$$
D_{3}(a, b)=\left\{\begin{array}{cc}
\frac{1}{6}(b-a)^{2}(b+2 a), & a>0, b>0 \\
\frac{1}{6}\left[b^{3}+a^{2}(3 b-2 a)\right], & a<0, b>0 \\
-\frac{1}{6}(b-a)^{2}(b+2 a), & a<0, b<0
\end{array}\right.
$$

We note that this result coincides with Corollary 2.6 in the special case a, $b>0$ [12].

Remark 2.7. If the mapping $\eta$ satisfies condition $C$ then using the inequality (2.2) in the proof of Theorem 2.5, the inequality (2.4) becomes to the following inequality:

$$
\begin{aligned}
& \left|f(a+\eta(b, a))[a+\eta(b, a)]-f(a) a-\int_{a}^{a+\eta(b, a)} f(x) d x\right| \\
& \leq \eta^{-\frac{1}{q}}(b, a) D_{1}^{1-\frac{1}{q}}(a, b)\left[\left|f^{\prime}(a+\eta(b, a))\right|^{q} D_{2}(a, b)+\left|f^{\prime}(a)\right|^{q} D_{3}(a, b)\right]^{\frac{1}{q}} .
\end{aligned}
$$

We note that by using of the preinvexity of $\left|f^{\prime}\right|^{q}$ we have $\left|f^{\prime}(a+\eta(b, a))\right|^{q} \leq\left|f^{\prime}(b)\right|^{q}$.Therefore, the inequality (2.5) is better than the inequality (2.4).

Corollary 2.8. If we take $q=1$ in Theorem 3 , then we have the following inequality:

$$
\left|f(a+\eta(b, a))[a+\eta(b, a)]-f(a) a-\int_{a}^{a+\eta(b, a)} f(x) d x\right| \leq \frac{1}{\eta(b, a)}\left[\left|f^{\prime}(b)\right| D_{2, \eta}(a, b)+\left|f^{\prime}(a)\right| D_{3, \eta}(a, b)\right]
$$

Theorem 2.9. Let $K \subseteq \mathbb{R}$ be an open invex subset with respect to mapping $\eta(\cdot, \cdot): K \times K \rightarrow \mathbb{R}^{n}$ and $a, b \in K$ with $\eta(b, a)>0$. Suppose that $f: K \rightarrow \mathbb{R}$ is a differentiable function on $K$ such that $f^{\prime} \in L[a, a+\eta(b, a)]$. If $\left|f^{\prime}\right|^{q}$ is preinvex on $K$ for $q>1$, then the following inequality holds:

$$
\left|f(a+\eta(b, a))[a+\eta(b, a)]-f(a) a-\int_{a}^{a+\eta(b, a)} f(x) d x\right| \leq \eta^{1-\frac{2}{q}}(b, a)\left[\left|f^{\prime}(b)\right|^{q} E_{1, \eta}(q, a, b)+\left|f^{\prime}(a)\right|^{q} E_{2, \eta}(q, a, b)\right]^{\frac{1}{q}}
$$


where

$$
E_{1, \eta}(q, a, b):=\left\{\begin{array}{lr}
\eta(b, a) L_{q+1}^{q+1}(a+\eta(b, a), a)-a \eta(b, a) L_{q}^{q}(a+\eta(b, a), a), & a>0, a+\eta(b, a)>0 \\
\eta(b, a) L_{q+1}^{q+1}(a+\eta(b, a),-a)-\frac{2 a}{q+1} A\left((a+\eta(b, a))^{q+1},(-a)^{q+1}\right), & a<0, a+\eta(b, a)>0 \\
\eta(b, a) L_{q+1}^{q+1}[-(a+\eta(b, a)),-a]+\frac{2 a}{q+1} A\left([-(a+\eta(b, a))]^{q+1},(-a)^{q+1}\right), & a<0, a+\eta(b, a)<0
\end{array}\right.
$$

and

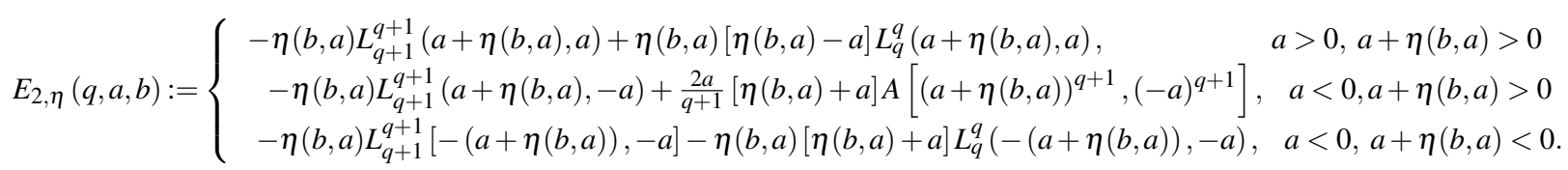

Proof. Since $\left|f^{\prime}\right|^{q}$ is preinvex on $[a, a+\eta(b, a)]$ for $q>1$, using Lemma 2.1 and the Hölder integral inequality, we have the following inequality:

$$
\begin{aligned}
& \left|f(a+\eta(b, a))[a+\eta(b, a)]-f(a) a-\int_{a}^{a+\eta(b, a)} f(x) d x\right| \\
& \leq \eta(b, a)\left(\int_{0}^{1} 1^{p} d x\right)^{\frac{1}{p}}\left(\int_{0}^{1}|a+t \eta(b, a)|^{q}\left|f^{\prime}(a+t \eta(b, a))\right|^{q} d x\right)^{\frac{1}{q}} \\
& =\eta(b, a)\left(\int_{0}^{1}|a+t \eta(b, a)|^{q}\left[t\left|f^{\prime}(b)\right|^{q}+(1-t)\left|f^{\prime}(a)\right|^{q}\right] d x\right)^{\frac{1}{q}} \\
& =\eta^{1-\frac{2}{q}}(b, a)\left(\left|f^{\prime}(b)\right|^{q} \int_{a}^{a+\eta(b, a)}(x-a)|x|^{q} d x+\left|f^{\prime}(a)\right|^{q} \int_{a}^{a+\eta(b, a)}[\eta(b, a)-x+a]|x|^{q} d x\right)^{\frac{1}{q}} \\
& =\eta^{1-\frac{2}{q}}(b, a)\left[\left|f^{\prime}(b)\right|^{q} E_{1, \eta}(q, a, b)+\left|f^{\prime}(a)\right|^{q} E_{2, \eta}(q, a, b)\right]^{\frac{1}{q}} .
\end{aligned}
$$

This completes the proof of theorem.

Corollary 2.10. Suppose that all the assumptions of Theorem 2.9 are satisfied. If we choose $\eta(b, a)=b-a$ then when $\left|f^{\prime}\right|^{q}$ is convex on $K$ for $q>1$ we have

$$
\left|\frac{f(b) b-f(a) a}{b-a}-\frac{1}{b-a} \int_{a}^{b} f(x) d x\right| \leq(b-a)^{-\frac{2}{q}}\left[\left|f^{\prime}(b)\right|^{q} E_{1}(q, a, b)+\left|f^{\prime}(a)\right|^{q} E_{2}(q, a, b)\right]^{\frac{1}{q}},
$$

where

$$
E_{1}(q, a, b)=\left\{\begin{array}{cc}
(b-a) L_{q+1}^{q+1}(b, a)-a(b-a) L_{q}^{q}(b, a), & a>0, b>0 \\
(b-a) L_{q+1}^{q+1}(b,-a)-\frac{2 a}{q+1} A\left(b^{q+1},(-a)^{q+1}\right), & a<0, b>0 \\
(b-a) L_{q+1}^{q+1}(-b,-a)+\frac{2 a}{q+1} A\left([-(b)]^{q+1},(-a)^{q+1}\right), a<0, b<0
\end{array}\right.
$$

and

$$
E_{2}(q, a, b)= \begin{cases}-(b-a)\left[L_{q+1}^{q+1}(b, a)-b L_{q}^{q}(b, a)\right], & a>0, b>0 \\ -(b-a) L_{q+1}^{q+1}(b,-a)+\frac{2 a b}{q+1} A\left(b^{q+1},(-a)^{q+1}\right), & a<0, b>0 \\ -(b-a)\left[L_{q+1}^{q+1}(-b,-a)+b L_{q}^{q}(-b,-a)\right], & a<0, b<0\end{cases}
$$

We note that this result coincides with Corollary 2.13 in the special case $a, b>0$ [12].

Remark 2.11. If the mapping $\eta$ satisfies condition $C$ then using the inequality (2.2) in the proof of Theorem 2.9, then the inequality (2.6) becomes to the following inequality:

$$
\left|f(a+\eta(b, a))[a+\eta(b, a)]-f(a) a-\int_{a}^{a+\eta(b, a)} f(x) d x\right| \leq \eta^{1-\frac{2}{q}}(b, a)\left[\mid f^{\prime}\left(\left.(a+\eta(b, a))\right|^{q} E_{1}(q, a, b)+\left|f^{\prime}(a)\right|^{q} E_{2}(q, a, b)\right]^{\frac{1}{q}}\right.
$$

Note that the inequality (2.7) is better than the inequality (2.6).

Theorem 2.12. Let $K \subseteq \mathbb{R}$ be an open invex subset with respect to mapping $\eta(\cdot, \cdot): K \times K \rightarrow \mathbb{R}^{n}$ and a,b $\in K$ with $\eta(b, a)>0$. Suppose that $f: K \rightarrow \mathbb{R}$ is a differentiable function on $K$ such that $f^{\prime} \in L[a, a+\eta(b, a)]$. If $\left|f^{\prime}\right|^{q}$ is preconcave on $K$ for $q>1$, then the following inequality holds:

$$
\left|f(a+\eta(b, a))[a+\eta(b, a)]-f(a) a-\int_{a}^{a+\eta(b, a)} f(x) d x\right| \leq \eta(b, a) F_{\eta}^{\frac{1}{p}}(p, a, b)\left|f^{\prime}\left(\frac{2 a+\eta(b, a)}{2}\right)\right|
$$

where

$$
F_{\eta}(p, a, b):=\left\{\begin{array}{cc}
L_{p}^{p}[a+\eta(b, a), a], & a>0, a+\eta(b, a)>0, \\
\frac{2 A\left((a+\eta(b, a))^{p+1},(-a)^{p+1}\right)}{(p+1) \eta(b, a)}, & a<0, a+\eta(b, a)>0, \\
L_{p}^{p}[-a,-(a+\eta(b, a))], & a<0, a+\eta(b, a)<0 .
\end{array}\right.
$$


Proof. Since $\left|f^{\prime}\right|^{q}$ is preconcave on $[a, a+\eta(b, a)]$ for $q>1$, with respect to Hermite-Hadamard inequality we get $\int_{a}^{b}\left|f^{\prime}(x)\right|^{q} d x \leq$ $\eta(b, a)\left|f^{\prime}\left(\frac{2 a+\eta(b, a)}{2}\right)\right|^{q}$. Using Lemma 2.1 and the Hölder integral inequality we have

$$
\begin{aligned}
\left|f(a+\eta(b, a))[a+\eta(b, a)]-f(a) a-\int_{a}^{a+\eta(b, a)} f(x) d x\right| & \leq \int_{a}^{a+\eta(b, a)}|x|\left|f^{\prime}(x)\right| d x \\
& \leq\left(\int_{a}^{a+\eta(b, a)}|x|^{p} d x\right)^{\frac{1}{p}}\left(\int_{a}^{a+\eta(b, a)}\left|f^{\prime}(x)\right|^{q} d x\right)^{\frac{1}{q}} \\
& =\eta(b, a)\left(\frac{1}{\eta(b, a)} \int_{a}^{a+\eta(b, a)}|x|^{p} d x\right)^{\frac{1}{p}}\left|f^{\prime}\left(\frac{2 a+\eta(b, a)}{2}\right)\right| \\
& =\eta(b, a) F_{\eta}^{\frac{1}{p}}(p, a, b)\left|f^{\prime}\left(\frac{2 a+\eta(b, a)}{2}\right)\right| .
\end{aligned}
$$

This completes the proof of theorem.

Corollary 2.13. Suppose that all the assumptions of Theorem 2.12 are satisfied. If we choose $\eta(b, a)=b-a$ then when $\left|f^{\prime}\right|^{q}$ is preconcave on $K$ for $q>1$ we have

$$
\left|\frac{f(b) b-f(a) a}{b-a}-\frac{1}{b-a} \int_{a}^{b} f(x) d x\right| \leq F^{\frac{1}{p}}(p, a, b)\left|f^{\prime}\left(\frac{a+b}{2}\right)\right|
$$

where

$$
F(p, a, b)= \begin{cases}L_{p}^{p}(b, a), & a>0, b>0 \\ \frac{2 A\left(b^{p+1},(-a)^{p+1}\right)}{{ }^{(p+1) \eta(b, a)},}, & a<0, b>0 \\ L_{p}^{p(-a,-b),} & a<0, b<0 .\end{cases}
$$

We note that this result coincides with Corollary 2.17 in the special case $a, b>0$ [12].

Now we will give new results for prequasiinvex functions by using Lemma 2.1 .

Theorem 2.14. Let $K \subseteq \mathbb{R}$ be an open invex subset with respect to mapping $\eta(\cdot, \cdot): K \times K \rightarrow \mathbb{R}^{n}$ and $a, b \in K$ with $\eta(b, a)>0$. Suppose that $f: K \rightarrow \mathbb{R}$ is a differentiable function on $K$ such that $f^{\prime} \in L[a, a+\eta(b, a)]$. If $\left|f^{\prime}\right|^{q}$ is prequasiinvex on $K$ for $q>1$, then the following inequality holds:

$$
\left|f(a+\eta(b, a))[a+\eta(b, a)]-f(a) a-\int_{a}^{a+\eta(b, a)} f(x) d x\right| \leq \eta(b, a) C_{\eta}(p, a, b)\left(\max \left\{\left|f^{\prime}(a)\right|^{q},\left|f^{\prime}(b)\right|^{q}\right\}\right)^{\frac{1}{q}}
$$

where

$$
C_{\eta}(p, a, b):= \begin{cases}L_{p}^{p}[a+\eta(b, a), a], & a>0, a+\eta(b, a)>0, \\ \frac{2 A\left[(a+\eta(b, a))^{p+1},(-a)^{p+1}\right]}{(p+1) \eta(b, a)}, & a<0, a+\eta(b, a)>0, \\ L_{p}^{p}[-a,-(a+\eta(b, a))], & a<0, a+\eta(b, a)<0 .\end{cases}
$$

Proof. If $\left|f^{\prime}\right|^{q}$ is prequasiinvex on $[a, a+\eta(b, a)]$ for $q>1$, using Lemma 2.1, the Hölder integral inequality and the inequality $\left|f^{\prime}(a+t \eta(b, a))\right|^{q} \leq$ $\max \left\{\left|f^{\prime}(a)\right|^{q},\left|f^{\prime}(b)\right|^{q}\right\}$ we have

$$
\begin{aligned}
\left|f(a+\eta(b, a))[a+\eta(b, a)]-f(a) a-\int_{a}^{a+\eta(b, a)} f(x) d x\right| & \leq \eta(b, a) \int_{0}^{1}|(a+t \eta(b, a))|\left|f^{\prime}(a+t \eta(b, a))\right| d t \\
& \leq \eta(b, a)\left(\int_{0}^{1}|a+t \eta(b, a)|^{p} d t\right)^{\frac{1}{p}}\left(\int_{0}^{1}\left|f^{\prime}(a+t \eta(b, a))\right|^{q} d t\right) \\
& \left.\leq \eta(b, a)\left(\int_{0}^{1}|a+t \eta(b, a)|^{p} d t\right)^{\frac{1}{q}}\left(\int_{0}^{1} \max \left\{\left|f^{\prime}(a)\right|^{q},\left|f^{\prime}(b)\right|^{q}\right\} d t\right)\right)^{\frac{1}{q}} \\
& =\eta(b, a)\left(\frac{1}{\eta(b, a)} \int_{a}^{a+\eta(b, a)}|x|^{p} d x\right)^{\frac{1}{p}}\left(\max \left\{\left|f^{\prime}(a)\right|^{q},\left|f^{\prime}(b)\right|^{q}\right\}\right)^{\frac{1}{q}} \\
& =\eta(b, a) C_{\eta}(p, a, b)\left(\max \left\{\left|f^{\prime}(a)\right|^{q},\left|f^{\prime}(b)\right|^{q}\right\}\right)^{\frac{1}{q}},
\end{aligned}
$$

This completes the proof of theorem.

Corollary 2.15. Suppose that all the assumptions of Theorem 2.14 are satisfied. If we choose $\eta(b, a)=b-a$ then when $\left|f^{\prime}\right|^{q}$ is prequasiinvex on $K$ for $q>1$ we have

$$
\left|\frac{f(b) b-f(a) a}{b-a}-\frac{1}{b-a} \int_{a}^{b} f(x) d x\right| \leq C(p, a, b)\left(\max \left\{\left|f^{\prime}(a)\right|^{q},\left|f^{\prime}(b)\right|^{q}\right\}\right)^{\frac{1}{q}}
$$


where

$$
C(p, a, b)= \begin{cases}L_{p}^{p}(b, a), & a>0, b>0, \\ \frac{2 A\left(b^{p+1},(-a)^{p+1}\right)}{(p+1)(b-a)}, & a<0, b>0, \\ L_{p}^{p}(-a,-b), & a<0, b<0 .\end{cases}
$$

We note that this result coincides with Corollary 2.1 in the special case $a, b>0$ [8].

Remark 2.16. If the mapping $\eta$ satisfies condition $C$ then by use of the prequasiinvexity of $\left|f^{\prime}\right|^{q}$ we get

$$
\begin{aligned}
\left|f^{\prime}(a+t \eta(b, a))\right|^{q} & =\left|f^{\prime}(a+\eta(b, a)+(1-t) \eta(a, a+\eta(b, a)))\right|^{q} \\
& \leq \max \left\{\left|f^{\prime}(a)\right|^{q},\left|f^{\prime}(a+\eta(b, a))\right|^{q}\right\}
\end{aligned}
$$

for every $t \in[0,1]$.

If we use the inequality (2.9) in the proof of Theorem 2.14, then the inequality (2.8) becomes to the following inequality:

$$
\left|f(a+\eta(b, a))[a+\eta(b, a)]-f(a) a-\int_{a}^{a+\eta(b, a)} f(x) d x\right| \leq \eta(b, a) C_{\eta}(p, a, b)\left(\max \left\{\left|f^{\prime}(a)\right|^{q},\left|f^{\prime}(a+\eta(b, a))\right|^{q}\right\}\right)^{\frac{1}{q}}
$$

We note that by use of the prequasiinvexity of $\left|f^{\prime}\right|^{q}$ we have $\left|f^{\prime}(a+\eta(b, a))\right|^{q} \leq \max \left\{\left|f^{\prime}(a)\right|^{q},\left|f^{\prime}(b)\right|^{q}\right\}$. Therefore, the inequality (2.10) is better than the inequality (2.8).

Theorem 2.17. Let $K \subseteq \mathbb{R}$ be an open invex subset with respect to mapping $\eta(\cdot, \cdot): K \times K \rightarrow \mathbb{R}^{n}$ and $a, b \in K$ with $\eta(b, a)>0$. Suppose that $f: K \rightarrow \mathbb{R}$ is a differentiable function on $K$ such that $f^{\prime} \in L[a, a+\eta(b, a)]$. If $\left|f^{\prime}\right|^{q}$ is prequasiinvex on $K$ for $q \geq 1$, then the following inequality holds:

$$
\left|f(a+\eta(b, a))[a+\eta(b, a)]-f(a) a-\int_{a}^{a+\eta(b, a)} f(x) d x\right| \leq\left[\max \left\{\left|f^{\prime}(a)\right|^{q},\left|f^{\prime}(b)\right|^{q}\right\}\right]^{\frac{1}{q}} D_{1, \eta}(a, b)
$$

where

$$
D_{1, \eta}(a, b):=\left\{\begin{array}{cc}
\eta(b, a) A(a, a+\eta(b, a)), & a>0, a+\eta(b, a)>0 \\
A\left(a^{2},[a+\eta(b, a)]^{2}\right), & a<0, a+\eta(b, a)>0 \\
-\eta(b, a) A(a, a+\eta(b, a)), & a<0, a+\eta(b, a)<0
\end{array} .\right.
$$

Proof. From Lemma 2.1 and Power-mean integral inequality, we obtain

$$
\begin{aligned}
& \left|f(a+\eta(b, a))[a+\eta(b, a)]-f(a) a-\int_{a}^{a+\eta(b, a)} f(x) d x\right| \\
& \leq \eta(b, a) \int_{0}^{1}|(a+t \eta(b, a))|\left|f^{\prime}(a+t \eta(b, a))\right| d t \\
& \leq \eta(b, a)\left(\int_{0}^{1}|a+t \eta(b, a)| d t\right)^{1-\frac{1}{q}}\left(\int_{0}^{1}|a+t \eta(b, a)|\left|f^{\prime}(a+t \eta(b, a))\right|^{q} d t\right)^{\frac{1}{q}} \\
& \leq \eta^{\frac{1}{q}}(b, a)\left(\int_{a}^{a+\eta(b, a)}|x| d x\right)^{1-\frac{1}{q}} \times\left(\int_{0}^{1}|a+t \eta(b, a)|\left[\max \left\{\left|f^{\prime}(a)\right|^{q},\left|f^{\prime}(b)\right|^{q}\right\}\right] d t\right)^{\frac{1}{q}} \\
& =\left[\max \left\{\left|f^{\prime}(a)\right|^{q},\left|f^{\prime}(b)\right|^{q}\right\}\right]^{\frac{1}{q}} \int_{a}^{a+\eta(b, a)}|x| d x \\
& =\left[\max \left\{\left|f^{\prime}(a)\right|^{q},\left|f^{\prime}(b)\right|^{q}\right\}\right]^{\frac{1}{q}} D_{1, \eta}(a, b) .
\end{aligned}
$$

This completes the proof of theorem.

Corollary 2.18. Suppose that all the assumptions of Theorem 2.17 are satisfied. If we choose $\eta(b, a)=b-a$ then when $\left|f^{\prime}\right|^{q}$ is prequasiinvex on $K$ for $q \geq 1$ we have

$$
\left|\frac{f(b) b-f(a) a-\int_{a}^{b} f(x) d x}{b-a}\right| \leq \frac{D_{1}(a, b)}{b-a}\left[\max \left\{\left|f^{\prime}(a)\right|^{q},\left|f^{\prime}(b)\right|^{q}\right\}\right]^{\frac{1}{q}}
$$

where

$$
D_{1}(a, b)=\left\{\begin{array}{cc}
(b-a) A(a, b), & a>0, b>0 \\
A\left(a^{2}, b^{2}\right), & a<0, b>0 \\
-(b-a) A(a, b), & a<0, b<0
\end{array} .\right.
$$

We note that this result coincides with Corollary 6 in the special case $a, b>0$ [8].

Remark 2.19. If we use the inequality (2.9) in the proof of Theorem 2.17, then the inequality (2.11) becomes the following inequality:

$$
\left|f(a+\eta(b, a))[a+\eta(b, a)]-f(a) a-\int_{a}^{a+\eta(b, a)} f(x) d x\right| \leq\left[\max \left\{\left|f^{\prime}(a)\right|^{q},\left|f^{\prime}(a+\eta(b, a))\right|^{q}\right\}\right]^{\frac{1}{q}} D_{1, \eta}(a, b)
$$

This inequality is better than the inequality (2.11). 
Corollary 2.20. If we take $q=1$ in Theorem 7 , then we have the following inequality:

$$
\left|f(a+\eta(b, a))[a+\eta(b, a)]-f(a) a-\int_{a}^{a+\eta(b, a)} f(x) d x\right| \leq \max \left\{\left|f^{\prime}(a)\right|,\left|f^{\prime}(b)\right|\right\} D_{1}(a, b)
$$

Theorem 2.21. Let $K \subseteq \mathbb{R}$ be an open invex subset with respect to mapping $\eta(\cdot, \cdot): K \times K \rightarrow \mathbb{R}^{n}$ and $a, b \in K$ with $\eta(b, a)>0$. Suppose that $f: K \rightarrow \mathbb{R}$ is a differentiable function on $K$ such that $f^{\prime} \in L[a, a+\eta(b, a)]$. If $\left|f^{\prime}\right|^{q}$ is prequasiinvex on $K$ for $q>1$, then the following inequality holds:

$$
\left|f(a+\eta(b, a))[a+\eta(b, a)]-f(a) a-\int_{a}^{a+\eta(b, a)} f(x) d x\right| \leq \eta^{1-\frac{1}{q}}(b, a)\left[\max \left\{\left|f^{\prime}(a)\right|^{q},\left|f^{\prime}(b)\right|^{q}\right\}\right]^{\frac{1}{q}} F_{1, \eta}^{\frac{1}{q}}(q, a, b)
$$

where

$$
F_{1, \eta}(p, a, b):=\left\{\begin{array}{lc}
\eta(b, a) L_{q}^{q}[a+\eta(b, a), a], & a>0, a+\eta(b, a)>0 \\
\frac{2}{q+1} A\left((a+\eta(b, a))^{p+1},(-a)^{p+1}\right), & a<0, a+\eta(b, a)>0 \\
\eta(b, a) L_{q}^{q}[-a,-(a+\eta(b, a))], & a<0, a+\eta(b, a)<0 .
\end{array}\right.
$$

Proof. Since $\left|f^{\prime}\right|^{q}$ for $q>1$ is prequasiinvex on $[a, b]$, using Lemma 2.1 and the Hölder integral inequality, we have the following inequality,

$$
\begin{aligned}
\left|f(a+\eta(b, a))[a+\eta(b, a)]-f(a) a-\int_{a}^{a+\eta(b, a)} f(x) d x\right| & \leq \eta(b, a)\left(\int_{0}^{1} 1^{p} d x\right)^{\frac{1}{p}}\left(\int_{0}^{1}|a+t \eta(b, a)|^{q}\left|f^{\prime}(a+t \eta(b, a))\right|^{q} d x\right)^{\frac{1}{q}} \\
& \leq \eta(b, a)\left(\int_{0}^{1}|a+t \eta(b, a)|^{q}\left[\max \left\{\left|f^{\prime}(a)\right|^{q},\left|f^{\prime}(b)\right|^{q}\right\}\right] d x\right)^{\frac{1}{q}} \\
& =\eta(b, a)\left[\max \left\{\left|f^{\prime}(a)\right|^{q},\left|f^{\prime}(b)\right|^{q}\right\}\right]^{\frac{1}{q}}\left(\int_{0}^{1}|a+t \eta(b, a)|^{q} d x\right)^{\frac{1}{q}} \\
& =\eta^{1-\frac{1}{q}}(b, a)\left[\max \left\{\left|f^{\prime}(a)\right|^{q},\left|f^{\prime}(b)\right|^{q}\right\}\right]^{\frac{1}{q}}\left(\int_{a}^{a+\eta(b, a)}|x|^{q} d t\right)^{\frac{1}{q}} \\
& =\eta^{1-\frac{1}{q}}(b, a)\left[\max \left\{\left|f^{\prime}(a)\right|^{q},\left|f^{\prime}(b)\right|^{q}\right\}\right]^{\frac{1}{q}} F_{1, \eta}^{\frac{1}{q}}(q, a, b),
\end{aligned}
$$

This completes the proof of theorem.

Corollary 2.22. Suppose that all the assumptions of Theorem 2.21 are satisfied. If we choose $\eta(b, a)=b-a$ then when $\left|f^{\prime}\right|^{q}$ is prequasiinvex on $K$ for $q>1$ we have

$$
\left|\frac{f(b) b-f(a) a}{b-a}-\frac{1}{b-a} \int_{a}^{b} f(x) d x\right| \leq(b-a)^{-\frac{1}{q}}\left[\max \left\{\left|f^{\prime}(a)\right|^{q},\left|f^{\prime}(b)\right|^{q}\right\}\right]^{\frac{1}{q}} F_{1}^{\frac{1}{q}}(q, a, b)
$$

where

$$
F_{1}(p, a, b)=\left\{\begin{array}{cc}
(b-a) L_{q}^{q}(b, a), & a>0, b>0, \\
\frac{2}{q+1} A\left(b^{p+1},(-a)^{p+1}\right), & a<0, b>0, \\
(b-a) L_{q}^{q}(-a,-b), & a<0, b<0 .
\end{array}\right.
$$

We note that this result coincides with Corollary 5 in the special case $a, b>0$ [8].

Remark 2.23. If we use the inequality (2.9) in the proof of Theorem 2.21, then the inequality (2.12) becomes the following inequality:

$$
\left|f(a+\eta(b, a))[a+\eta(b, a)]-f(a) a-\int_{a}^{a+\eta(b, a)} f(x) d x\right| \leq \eta^{1-\frac{1}{q}}(b, a)\left[\max \left\{\left|f^{\prime}(a)\right|^{q},\left|f^{\prime}(a+\eta(b, a))\right|^{q}\right\}\right]^{\frac{1}{q}} F_{1, \eta}^{\frac{1}{q}}(q, a, b)
$$

This inequality is better than the inequality (2.12).

\section{References}

[1] T. Antczak, Mean value in invexity analysis, Nonl. Anal. 60(2005), 1473-1484.

[2] A. Barani, A.G. Ghazanfari, S.S. Dragomir, Hermite-Hadamard inequality through prequasiinvex functions, RGMIA Research Report Collection, 14(2011), Article 48, 7 pp.
A. Barani, A.G. Ghazanfari, S.S. Dragomir, Hermite-Hadamard inequality for functions whose derivatives absolute values are preinvex, J. Inequal. 3] Aarani, A.G. Ghazanfa
Appl. 2012, 2012:247.

[4] S.S. Dragomir and C.E.M. Pearce, Selected Topics on Hermite-Hadamard Inequalities and Applications, RGMIA Monographs, Victoria University, 2000.

[5] J. Hadamard, Étude sur les propriétés des fonctions entières et en particulier d'une fonction considerée par Riemann, J. Math Pures Appl., 58 (1893), $171-215$.

[6] D. A. Ion, Some estimates on the Hermite-Hadamard inequality through quasi-convex functions, Annals of University of Craiova, Math. Comp. Sci. Ser. Volume 34, 2007, Pages 82-87.

[7] A. B. Israel and B. Mond, What is invexity? J. Aust. Math. Soc. Ser. B 28(1), 1-9 (1986).

[8] İ. İşcan, H. Kadakal and M. Kadakal, Some New Integral Inequalities for n-Times Differentiable Quasi-Convex Functions, Sigma Journal of Engineering and Natural Sciences, 35 (3), 363-368, 2017.

[9] M. Kadakal, Hermite-Hadamard and Simpson type inequalities for multiplicatively harmonically P-functions, Sigma Journal of Engineering and Natural Sciences, 37(4), (2019), 1311-1320. 
[10] H. Kadakal, M. Kadakal and İ. İşcan, Some New Integral Inequalities for n-Times Differentiable r-Convex and r-Concave Functions, Miskolc Mathematical Notes, 20(2) (2019), 997-1011.

[11] M. A. Latif and S. S. Dragomir, Some Hermite-Hadamard type inequalities for functions whose partial derivatives in absloute value are preinvex on the co-oordinates, Facta Universitatis (NIŠ) Ser. Math. Inform. Vol. 28, No 3 (2013), 257-270.

[12] S. Maden, H. Kadakal, M. Kadakal and İ. İşcan, Some new integral inequalities for n-times differentiable convex functions, Journal of Nonlinear Sciences and Applications, 10 (12), (2017), 6141-6148.

[13] M. Matloka, On some new inequalities for differentiable $\left(h_{1} ; h_{2}\right)$-preinvex functions on the co-ordinates, Mathematics and Statistics 2(1), (2014), 6-14.

[14] S.R. Mohan, S.K. Neogy, On invex sets and preinvex functions, J. Math. Anal. Appl., 189 (1995), 901-908.

[15] M. A. Noor, Hermite-Hadamard integral inequalities for log-preinvex functions, J. Math. Anal. Approx. Theory 2 (2007) 126-131.

[16] M.A. Noor, Invex equilibrium problems. J. Math. Anal. Appl. 302, 463-475 (2005).

[17] M.A. Noor, Variational-like inequalities. Optimization 30, 323-330 (1994).

[18] S. Özcan, Some Integral Inequalities for Harmonically $(\alpha, s)$-Convex Functions, Journal of Function Spaces, 2019, (2019) Article ID 2394021, 8 pages.

[19] S. Özcan, On Refinements of Some Integral Inequalities for Differentiable Prequasiinvex Functions, Filomat, 33(14), (2019), 4377-4385.

[20] S. Özcan, Some Integral Inequalities of Hermite-Hadamard Type for Multiplicatively Preinvex Functions, AIMS Mathematics, 5(2), (2020), 1505-1518.

[21] S. Özcan and İ. İşcan, Some new Hermite-Hadamard type inequalities for s-convex functions and their applications, Journal of Inequalities and Applications 2019(201), (2019).

[22] J.E. Pečarić, F. Porschan and Y. L. Tong, Convex Functions, Partial Orderings, and Statistical Applications, Academic Press Inc., 1992.

[23] R. Pini, Invexity and generalized convexity. Optimization 22, 513-525 (1991).

[24] T. Toplu, İ. İşcan and M. Kadakal, On n-polinomial convexity and some related inequalities, Aims Mathematics, 5(2) (2020), $1304-1318$.

[25] T. Weir and B. Mond, Preinvex functions in multiple objective optimization, Journal of Mathematical Analysis and Applications, 136 (1998) $29-38$.

[26] X. M. Yang, X. Q. Yang, K. L. Teo, Generalized invexity and generalized invariant monotonicity, J. Optim. Theory. Appl., 117(2003), 607-625.

[27] X.M.Yang and D. Li, On properties of preinvex functions. J. Math. Anal. Appl. 256, 229-241 (2001). 